\title{
DEVELOPMENT AND APPLICATION OF A TEST MIXTURE FOR UNTARGETED LIQUID CHROMATOGRAPHY-MASS SPECTROMETRY ANALYSIS OF URINE SAMPLES
}

\author{
Clarisse L. Torres ${ }^{\mathrm{a}}$, Vinicius F. Sardela ${ }^{\mathrm{a}}$, Fernanda B. Scalco ${ }^{\mathrm{a}}$, Francisco R. de Aquino Neto ${ }^{\mathrm{a}}$ and Rafael Garrett ${ }^{\mathrm{a} *}$,(1) \\ anstituto de Química, Universidade Federal do Rio de Janeiro, Ilha do Fundão, 21941-916 Rio Janeiro - RJ - Brasil
}

Recebido em 06/04/2021; aceito em 05/07/2021; publicado na web em 23/07/2021

\begin{abstract}
Metabolic profiling of complex biological matrices based on liquid chromatography-mass spectrometry (LC-MS) allows detecting a wide range of metabolites with distinct concentrations and physicochemical properties. Given the complexity of samples and the necessity of a comprehensive approach in untargeted metabolomics, quality control strategies are mandatory to obtain high-quality data. The LC-MS performance must be monitored and evaluated to guarantee data reliability. In this study, a test mixture (TM) was developed, systematically evaluated, and applied to untargeted metabolomics of urine samples from individuals suspected of inborn errors of metabolism. The TM was composed of fifteen analytes that eluted across the entire gradient in reversed-phase columns and ionized in positive/negative electrospray modes. It helped set the LC-MS conditions for urine analysis, from sample reconstitution solvent to selecting the MS ion source parameters. The TM quickly indicated column stationary phase degradation during the batch analysis when employed to monitor and evaluate the LC-MS system in an untargeted metabolomic analysis. Thus, in addition to pooled QC samples, a TM can be employed in untargeted metabolomics to rapidly assess the system performance avoiding unnecessary efforts for further data treatment and multivariate analysis of poor-quality data.
\end{abstract}

Keywords: LC-MS; untargeted metabolomics; quality control; IEM.

\section{INTRODUCTION}

Untargeted metabolomics has proven its great potential as a global approach in metabolic profiling of complex biological matrices to improve diagnostic sensitivity and identify new disease's specific biomarkers in clinical applications. ${ }^{1}$ Untargeted assays aim to simultaneously measure as many metabolites as possible from biological samples without bias. ${ }^{2}$ However, there are still sources of errors regarding the metabolomics workflow that should be minimized to obtain high-quality data. ${ }^{3-9}$

Liquid chromatography-mass spectrometry (LC-MS) is a commonly used technique in untargeted metabolomic studies. Hence, the LC-MS system's performance must be evaluated and monitored to guarantee data reliability, as variations in instrumentation are expected due to column degradation, matrix effects, sample carryover, among others. For example, Brunius et al. ${ }^{10}$ described that data generated from multiple batches during large-scale untargeted metabolomics were affected by measurement errors inherent to alterations in signal intensity, drift in mass accuracy, and retention times between samples from both within and between batches.

Quality assurance (QA) and quality control (QC) protocols are essential when an untargeted approach is used, as "unknown features" (previous unknown metabolites) can be further identified as potential biological markers after data processing. ${ }^{11}$ Poor data quality may result in false discoveries without biological relevance, leading to an erroneous hypothesis. ${ }^{12}$ Therefore, before and during the analysis of any biological sample, the suitability of a given analytical platform should be assessed, and thus its analytical performance assured.

Currently, the approaches used to ensure the validity of untargeted metabolomic data are mainly focused on adopting system suitability procedures and the use of pooled QC samples as strategies for monitoring and evaluating the analytical system robustness. However, no community-agreed acceptance criteria or global standards

*e-mail: rafael_garrett@iq.ufrj.br procedures have yet been achieved, reinforcing the utmost importance of describing the QC procedures used in the studies to guarantee the quality of the data obtained. In the last years, a great effort has been made to educate the metabolomics community to apply QA and QC procedures. Protocols and guidelines regarding this relevant topic have been published in the literature ${ }^{4,8,9,11,13,14}$ and the Metabolomics Quality Assurance and Quality Control Consortium (mQACC) has been created to address the current challenges in QA and QC in untargeted metabolomic studies. ${ }^{15}$ Most of these protocols and guidelines have focused on adopting, evaluating, and proper use of pooled QC samples to measure the analytical system's performance and the quality of data obtained. Test mixtures (TMs) are highlighted as important for system suitability and a rapid evaluation of data before statistical analysis. However, as far as we know, a systematic evaluation of a TM in LC-MS untargeted metabolomics for urine analysis has not yet been addressed.

TMs are solutions of a limited number of analytical standards employed to evaluate an analytical method or equipment's performance. They can be purchased from vendors or tailored by users. ${ }^{4,9,11,16,17}$ However, despite its importance, it seems that TMs are still neglected, or at least not described, in many LC-MS based untargeted metabolomic studies. Reproducible results are imperative to obtain reliable metabolic profile data. In this context, using a TM before and after batches of samples is an important strategy for the rapid assessment of the LC-MS systems, especially when automatic data reports can be generated by the LC-MS controlling software.

In addition to pooled QC samples, TMs can rapidly determine if the instrumental setup is suitable for the analysis and determine if significant changes have occurred during the batch. Changes in chromatographic separation performance, detector response, and mass accuracy are thus recorded, allowing the analyst to do troubleshooting and suggest the corrective maintenance to be performed., ${ }^{5,9}$ The TM data assure that it is worth proceeding to evaluate the results from the pooled QC samples. Besides, data recorded in long-term studies by the same method enables the use of control charts to track the analytical system's behavior and tendencies. 
In this work, a TM solution of fifteen analytical standards with different interactions with reversed-phase $\mathrm{C}_{18}$ columns and ionization in positive/negative electrospray-MS modes was developed, systematically evaluated, and applied to an untargeted metabolomics study of urine samples from individuals suspected of inborn errors of metabolism (IEMs) as a proof of concept.

IEMs are inherited genetic diseases caused by mutations in genes coding for proteins that function in metabolism. ${ }^{18}$ These mutations result in inactive or partially active proteins, which may alter the synthesis, transport, degradation, or storage of molecules in the organism. ${ }^{19}$ There are more than 1.000 IEM described so far, ${ }^{20}$ and when the diagnosis can be performed by mass spectrometry, targeted methods are traditionally employed.$^{21}$ However, untargeted metabolomics can detect a much more comprehensive range of metabolites than targeted approaches, expanding our knowledge about new biomarkers and metabolic pathways involved in those diseases. ${ }^{22,23}$

\section{EXPERIMENTAL}

\section{Materials and reagents}

Acetonitrile (ACN, HPLC/Spectro grade) and formic acid (FA, LC-MS grade) were from Tedia (Fairfield, USA). All the analytical standards (purity $\geq 95 \%$ ) were from Sigma-Aldrich (São Paulo, Brazil). High-purity water $(18.2 \mathrm{M} \Omega \cdot \mathrm{cm})$ was from a Millipore Milli-Q purification system (Billerica, MA, USA). LC columns Hypersil Gold $\mathrm{C}_{18}(100 \mathrm{~mm} \times 2.1 \mathrm{~mm}, 1.9 \mu \mathrm{m})$ and Syncronis $\mathrm{C}_{18}$ (50 $\mathrm{mm} \times 2.1 \mathrm{~mm}, 1.7 \mu \mathrm{m})$ were from Thermo Scientific.

\section{The TM solution}

The TM composition is shown in Table 1. It included analytical standards commonly found in urine (e.g., hippuric acid) or similar to compounds found in urine (e.g., 3,5-dinitrobenzoic acid) or that may be found in urine samples (e.g., miconazole). It was prepared by adding $10 \mu \mathrm{L}$ of stocked solutions $\left(1.0 \mathrm{mg} \mathrm{mL}^{-1}\right.$ in $\left.\mathrm{MeOH}\right)$ of each analyte in water:ACN 85:15 (v/v) containing $0.1 \%$ of formic acid to obtain final concentrations of $200 \mathrm{ng} \mathrm{mL}^{-1}$. Nonetheless, different proportions of water:ACN (95:5, 85:15, 75:25, and 50:50\%; $\mathrm{v} / \mathrm{v})$ were tested as a reconstitution solvent to obtain a proper peak shape of the polar substances along with the recovery of less polar ones. Peak symmetry, retention times $\left(t_{R}\right)$, resolution between isobaric substances, and experimental $\mathrm{m} / \mathrm{z}$ values were used to assess chromatographic stability and MS mass accuracy.

\section{LC-HRMS analysis}

The LC separation was performed in a Dionex UltiMate 3000 UHPLC system (Thermo Fisher Scientific, Bremen, Germany) using reversed-phase columns at $40^{\circ} \mathrm{C}$. The mobile phases consisted of (A) water and (B) ACN, both with $0.1 \%$ of formic acid and a flow rate of $350 \mu \mathrm{L} \mathrm{min}{ }^{-1}$. The elution gradient was 0 to $1 \mathrm{~min}, 5 \% \mathrm{~B}$; 1 to $9 \mathrm{~min}, 5-60 \% \mathrm{~B} ; 9$ to $13 \mathrm{~min}, 60-95 \% \mathrm{~B} ; 13$ to $16 \mathrm{~min}, 95 \% \mathrm{~B}$ (column washing); 16.1 to $20.0 \mathrm{~min}, 5 \% \mathrm{~B}$ (column equilibration to the initial conditions). The overall run time was $20 \mathrm{~min}$, and the injection volume $3 \mu \mathrm{L}$. The LC effluent was pumped to a Q-Exactive Plus Orbitrap mass spectrometer (Thermo Fisher Scientific, Bremen, Germany) equipped with an ESI source operating by switching between positive $(+)$ and negative (-) ionization modes. Three levels of the S-Lens RF voltages (50, 60, and 70 a.u., arbitrary units), two capillary temperatures $\left(300\right.$ and $320^{\circ} \mathrm{C}$ ), and spray voltage values (3.9 and $3.4 \mathrm{kV}$ for ESI+, 3.6 and $3.1 \mathrm{kV}$ for ESI-) were evaluated. They represented common values employed in our laboratory and metabolomics studies using the QExactive mass spectrometer. ${ }^{24-29}$ The sheath and auxiliary gas flow rates (both nitrogen) were set to 45 and 20 (a.u.), respectively.

\section{Column performance evaluation}

In addition to a preliminary analysis of the TM solution in the Hypersil Gold column $(100 \mathrm{~mm} \times 2.1 \mathrm{~mm}, 1.9 \mu \mathrm{m})$, the performance of six extensively used columns (Syncronis $\mathrm{C}_{18} 50 \mathrm{~mm} \times 2.1 \mathrm{~mm}$, $1.7 \mu \mathrm{m}$; with at least 350 injections each) were evaluated and compared to those results obtained by a brand-new one (all of them with the same batch number). The analysis was done by injecting $3 \mu \mathrm{L}$ of the TM solution at $200 \mathrm{ng} \mathrm{mL}^{-1}$. The analytes' $t_{R}$, peak areas, and peak shapes were assessed using an automated data processing in

Table 1. Analytical standards used in the preparation of the TM solution, their respective retention times, ionization mode chosen for TM evaluation and monitoring ions by LC-MS analysis using a Hypersil Gold Column $\left(\right.$ RP-C $\left._{18} 100 \mathrm{~mm} \times 2.1 \mathrm{~mm}, 1.9 \mu \mathrm{m}\right)$

\begin{tabular}{lccccc}
\hline $\mathrm{N}$ & Analytical standards & Chemical formula & $\mathrm{t}_{\mathrm{R}}(\mathrm{min})$ & ESI mode & Monitoring ion $(\mathrm{m} / z)$ \\
\hline $\mathbf{1}$ & Quinic acid & $\mathrm{C}_{7} \mathrm{H}_{12} \mathrm{O}_{6}$ & 0.79 & {$[\mathrm{M}-\mathrm{H}]^{-}$} & 191.0561 \\
$\mathbf{2}$ & Heptaminol & $\mathrm{C}_{8} \mathrm{H}_{19} \mathrm{NO}$ & 1.42 & {$[\mathrm{M}+\mathrm{H}]^{+}$} & 146.1539 \\
$\mathbf{3}$ & Benzamide & $\mathrm{C}_{7} \mathrm{H}_{7} \mathrm{NO}$ & 3.24 & {$[\mathrm{M}+\mathrm{H}]^{+}$} & 122.0600 \\
$\mathbf{4}$ & Hippuric acid & $\mathrm{C}_{9} \mathrm{H}_{9} \mathrm{NO}_{3}$ & 3.81 & {$[\mathrm{M}-\mathrm{H}]^{-}$} & 178.0510 \\
$\mathbf{5}$ & Chlorogenic acid & $\mathrm{C}_{16} \mathrm{H}_{18} \mathrm{O}_{9}$ & 3.85 & {$[\mathrm{M}-\mathrm{H}]^{-}$} & 353.0878 \\
$\mathbf{6}$ & Caffeine & $\mathrm{C}_{8} \mathrm{H}_{10} \mathrm{~N}_{4} \mathrm{O}_{2}$ & 4.22 & {$[\mathrm{M}+\mathrm{H}]^{+}$} & 195.0877 \\
$\mathbf{7}$ & Propylhexedrine & $\mathrm{C}_{10} \mathrm{H}_{21} \mathrm{~N}$ & 7.15 & {$[\mathrm{M}+\mathrm{H}]^{+}$} & 156.1747 \\
$\mathbf{8}$ & Octan-2-amine & $\mathrm{C}_{8} \mathrm{H}_{19} \mathrm{~N}$ & 7.17 & {$[\mathrm{M}+\mathrm{H}]^{+}$} & 130.1590 \\
$\mathbf{9}$ & 3,5 -dinitrobenzoic acid & $\mathrm{C}_{7} \mathrm{H}_{4} \mathrm{O}_{6} \mathrm{~N}_{2}$ & {$[\mathrm{M}-\mathrm{H}]^{-}$} & 210.9997 \\
$\mathbf{1 0}$ & Triamcinolone & $\mathrm{C}_{21} \mathrm{H}_{27} \mathrm{FO}_{6}$ & 7.38 & {$[\mathrm{M}+\mathrm{H}]^{+}$} & 393.1719 \\
$\mathbf{1 1}$ & Octan-1-amine & $\mathrm{C}_{8} \mathrm{H}_{19} \mathrm{~N}$ & 7.38 & {$[\mathrm{M}-\mathrm{H}]^{-}$} & 130.1590 \\
$\mathbf{1 2}$ & Furosemide & $\mathrm{C}_{12} \mathrm{H}_{11} \mathrm{ClN}_{2} \mathrm{O}_{5} \mathrm{~S}$ & 7.71 & 8.53 & 329.0004 \\
$\mathbf{1 3}$ & Benzophenone & $\mathrm{C}_{13} \mathrm{H}_{10} \mathrm{O}$ & 10.97 & {$[\mathrm{M}+\mathrm{H}]^{+}$} & 183.0804 \\
$\mathbf{1 4}$ & Miconazole & $\mathrm{C}_{18} \mathrm{H}_{14} \mathrm{Cl}_{4} \mathrm{~N}_{2} \mathrm{O}$ & 11.06 & {$[\mathrm{M}+\mathrm{H}]^{+}$} & 414.9933 \\
$\mathbf{1 5}$ & Bromantane & $\mathrm{C}_{16} \mathrm{H}_{20} \mathrm{BrN}^{+}$ & 14.70 & {$[\mathrm{M}+\mathrm{H}]^{+}$} & 306.0852 \\
\hline
\end{tabular}

$\mathrm{t}_{\mathrm{R}}=$ retention time, $\mathrm{ESI}=$ electrospray ionization. 
the TraceFinder Forensics software v3.2 (Thermo Fisher Scientific, Bremen, Germany).

\section{Untargeted metabolomics of urine samples from individuals suspected of IEM}

The TM solution was employed in an untargeted metabolomics study of urine samples as a proof of concept to provide a rapid examination of the LC-MS system performance and assessment of data quality. Fifty urine samples, being 25 from healthy volunteers and 25 from individuals suspected of having IEM, were used (samples submitted by doctors to routine analysis at the Laboratório de Erros Inatos do Metabolismo-LABEIM, LADETEC-IQ/UFRJ, Brazil). This study was approved by the Institutional Ethics Committee, Clementino Fraga Filho University Hospital-Federal University of Rio de Janeiro (HUCFF-UFRJ - number 3120923).

Samples were prepared by diluting $300 \mu \mathrm{L}$ of the urines to $1.2 \mathrm{~mL}$ of solvent MeOH:ACN $(1: 1, v / v)$ in a centrifuge tube. The solutions were vortexed for 15 seconds, incubated at $4{ }^{\circ} \mathrm{C}$ for $15 \mathrm{~min}$, and centrifuged at $10,000 \mathrm{x}$ g for $15 \mathrm{~min}$. Then, $300 \mu \mathrm{L}$ of the supernatant were transferred, and the solvent evaporated to dryness under a nitrogen stream in a water bath at $40^{\circ} \mathrm{C}$. The dried extracts were stored at $-20^{\circ} \mathrm{C}$ until further analysis. A pooled QC sample was prepared by mixing $10 \mu \mathrm{L}$ of each sample before injection.

Dried samples were reconstituted using $300 \mu \mathrm{L}$ of water:ACN $85: 15 \%(\mathrm{v} / \mathrm{v})$ containing $0.1 \%$ formic acid and injected into the LC-MS system. The pooled QC sample was injected periodically during the batch analysis. The sequence of analyses was set up according to the following injection order: mobile phase blank $>$ $\mathrm{TM}>5$ pooled QC samples (conditioning step) $>\mathrm{TM}>5$ pooled QC samples $>10$ randomized test samples $>$ pooled QC sample. The cycle of injecting 10 randomized test samples followed by the pooled QC sample was repeated until the last test sample was analyzed. Then, the TM was reinjected at the end of the batch.

This untargeted metabolomic analysis was performed in two other Syncronis RP-C ${ }_{18}$ columns, one with a low number of injected samples and the other extensively used (identified here as $\mathrm{C} 07$ and C08, respectively).

\section{Data processing and chemometric analysis}

Data processing, such as peak picking, deconvolution, and alignment were performed in the MS-Dial software version 3.98. ${ }^{30}$ The parameters used were MS1 and MS2 tolerances of 0.005 and
0.05 , respectively; minimum peak height of 500,000; mass slice width of $0.05 \mathrm{Da}$; linear-weighted moving average as the smoothing method using 3 scans and peak width of 5 scans; sigma window value for deconvolution of $0.4 ; 0.1 \mathrm{~min}$ and 0.005 Da tolerance for peak alignment.

The aligned data table from the MS-DIAL was exported as .txt file, converted to .csv, and uploaded to the Metaboanalyst platform ${ }^{31}$ to perform the multivariate data analysis. Data were normalized by the sum, Pareto scaled, and submitted to Principal Component Analysis (PCA) and Partial least squares-discriminant analysis (PLS-DA).

\section{RESULTS AND DISCUSSION}

\section{The TM solution}

The TM solution included polyhydroxylated compounds, neutrals, basic and acidic functions ( $\log \mathrm{P}$ values ranged from -2.4 to 5.0), allowing the evaluation of chromatographic parameters, such as peak shape and adsorption effects. Besides, compounds in the TM could be analyzed by ESI+ and/or ESI- ionization modes (see supplementary Figure $1 \mathrm{~S}$ for the chemical structures and $\log \mathrm{P}$ values). The selection of compounds was based on requirements, such as (i) the analytical performance of the LC-MS system could be evaluated in a single chromatographic analysis with compounds ionizing in both ionization modes; (ii) compounds should be of easy access and at a reasonable price; (iii) compounds should resemble those found in urine samples; (iv) and the TM should give all the information required to determine interventions at the system (i.e., MS cleaning procedure, calibration or column change). The extracted ion chromatograms for each compound of the TM solution in the Hypersil Gold column $(100 \mathrm{~mm} \times 2.1 \mathrm{~mm}, 1.9 \mu \mathrm{m})$ are shown in Figure 1 .

Some of the analytes shown in Figure 1 are typically found in urine samples. Hippuric acid, for example, is a common endogenous compound. Caffeine, chlorogenic acid, and quinic acid are related to food intake and frequently detected in urine samples. Other substances, like the alkylamines, miconazole, and triamcinolone are medicines. They might be found in the urine of individuals who have used these drugs.

The elution order of the compounds reflected their interactions with the sorbent and the mobile phase. At the beginning of the chromatographic run, the small structures with hydroxy groups (heptaminol (1) and quinic acid (2)) were the first to be eluted (Figure 1). This behavior was expected as the interactions of these compounds with the stationary phase involved dipole-induced dipole
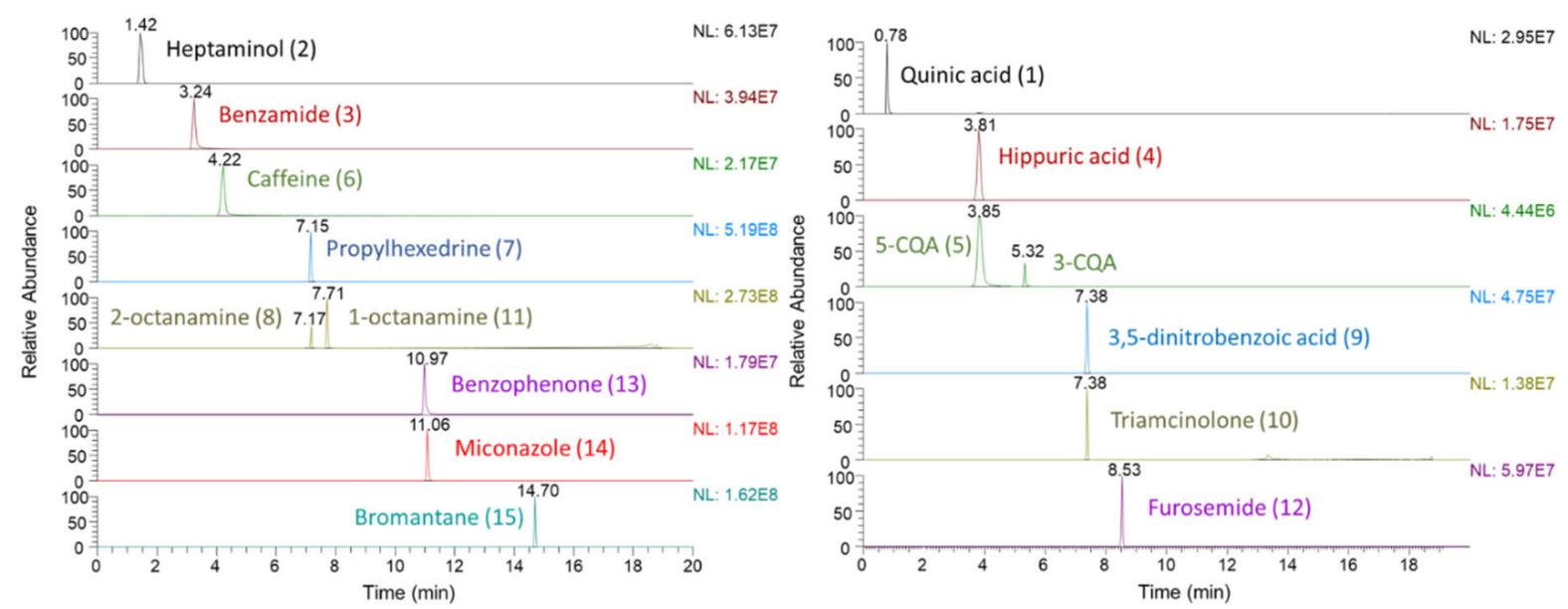

Figure 1. Typical data acquired for the TM solution using a RP-C ${ }_{18}$ Hypersil Gold column (100 mm $\left.\times 2.1 \mathrm{~mm}, 1.9 \mu \mathrm{m}\right)$. Compounds number 2, 3, 6, 7, 8, 11, 13, 14, 15 were from the ESI+ analysis (left), while compounds 1, 4, 5, 9, 10, 12 were from the ESI- analysis (right) 
force. In contrast, their interactions with the mobile phase (mostly water at this LC gradient stage) involved hydrogen bond interactions, being this last one the strongest.

Following the elution of compounds (1) and (2), it was observed the elution of other functionalized but aromatic compounds (e.g., benzamide (3), hippuric acid (4), and caffeine (6)) that better interacted with the $\mathrm{C}_{18}$ column through dipole-induced dipole than compounds (1) and (2). In the middle of the run were eluted the amino compounds (propylhexedrine (7), 2-octanamine (8), and 1-octanamine (11)) with long hydrocarbon chains (Figure 1). Here, the retention was primarily due to van der Waals interactions between the analytes and the sorbent because of the preferred interaction of the hydrocarbon chain with the stationary phase. The two alkylamines isomers, with molecular formula $\mathrm{C}_{8} \mathrm{H}_{18} \mathrm{~N}$ (2-octanamine (8) and 1-octanamine (11)) were chosen to assess the resolution power of the separation method as degradation of stationary phase, which may expose active acid centers (e.g., silanol groups) resulting in strong acid-based interaction, can lead to their co-elution due to peak broadening and tailing.

Compounds 3,5-dinitrobenzoic acid (9) and triamcinolone (10) eluted at the same $t_{\mathrm{R}}$. However, they could be easily differentiated by their different molecular masses. At the end of the run, the elution of the less polar and functionalized compounds (e.g., bromantane (15)) was observed due to major hydrophobic interactions with the stationary phase.

All the analytes showed a symmetrical peak shape along the LC run with no evidence of peak splitting or broadening (Figure 1). The $\mathrm{m} / \mathrm{z}$ error was below $5.0 \mathrm{ppm}$ compared to the theoretical values.

Commercially available TM solutions or those described in the literature generally contain 4-8 standards employed to assess the

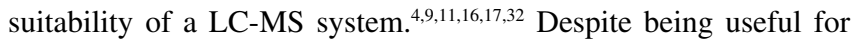
their purpose, they are in general mixtures of generic compounds, which limit their utility only for specific assays. Differently, the TM solution proposed here was tailor-made for system suitability in untargeted methods, particularly for urine analysis. Its more comprehensive range of compounds with distinct adsorptive effects in reverse-phase separation mode, molecular masses, and resemblance to those compounds found in urine samples may better reflect the chromatographic behavior of urinary metabolites. Furthermore, the TM covered many types of peak distortions that are important to understand the chromatographic process, such as peak broadening, peak tailing, adsorption effects, and distorted peaks.

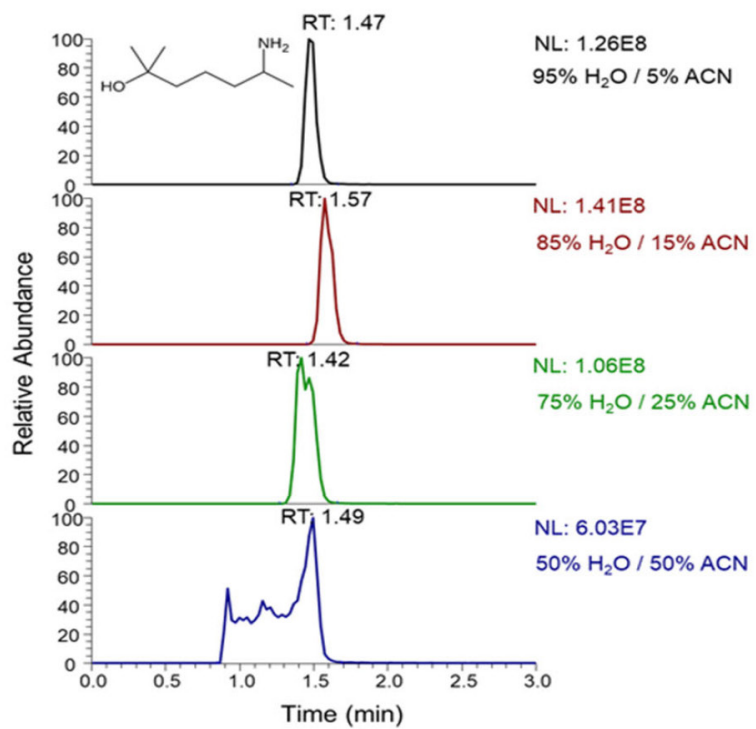

\section{Evaluation of sample reconstitution solvents}

In RP- $\mathrm{C}_{18}$ columns, substances with $\mathrm{t}_{\mathrm{R}}$ around the column void volume are greatly affected by the reconstitution solvent. An adequate solvent should be quite similar to the initial mobile phase of the chromatographic run to avoid compound dispersion and initial band broadening, which will result in peak broadening.

Different proportions of water:ACN $(95: 5,85: 15,75: 25$, and $50: 50 \% ; \mathrm{v} / \mathrm{v})$ were evaluated to obtain Gaussian-like peaks without peak fronting, tailing, or splitting of the substances present in the TM as a way to help in the selection of the best reconstitution solvent for the urine samples. The extracted ion chromatograms for heptaminol (2) and bromantane (15) using different proportions of water:ACN are shown in Figure 2.

Compounds eluted at the beginning of the chromatographic run can be drastically affected by the solvent composition due to the heterogeneous initial distribution of the analytes. In the case of heptaminol, which strongly interacts with water-rich solvents by hydrogen bonding, it was observed a distortion in peak shape (peak splitting) when the reconstitution solvent proportions were water:ACN $75: 25$ or $50: 50 \%(\mathrm{v} / \mathrm{v})$. As the concentration of ACN increased, the compound distribution between the mobile and stationary phases became more diffuse than water:ACN 95:5\% because of the increased penetration of the $\mathrm{ACN}$ into the chains of the $\mathrm{C}_{18}$, resulting in a non-homogeneous distribution of this analyte. The combination of 95:5\% water:ACN showed the best peak shape for heptaminol. However, this water proportion did not allow the proper solubilization of compounds that are mostly soluble in organic solvents, represented here by the bromantane, the last peak to be eluted in the TM solution. This would limit the detection of substances with similar physicochemical properties. For example, the signal intensity of bromantane in 50:50\% water:ACN was ca. 4 times higher than in 95:5\% water:ACN. Therefore, by choosing an appropriate condition (water:ACN 85:15\% solution), all peaks presented the desired Gaussian-like shape and high intensity. The heptaminol peak was almost perfectly shaped, showing a slight distortion that set the limits to control any unfavorable condition.

\section{MS ion source tuning using the TM}

The TM was employed to perform the first tune of the mass

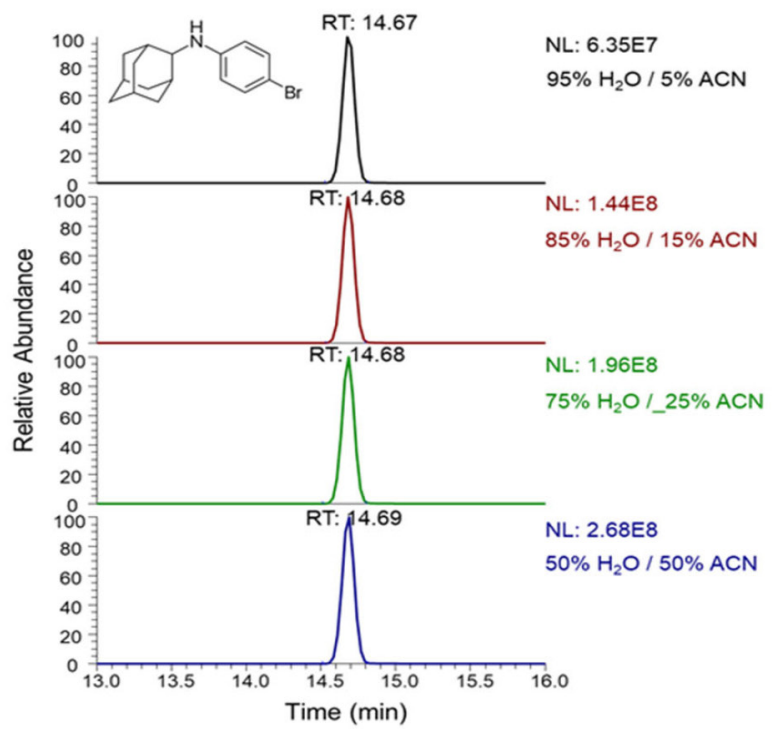

Figure 2. Extracted ion chromatograms for heptaminol (left) and bromantane (right) in the Hypersil Gold column using different reconstitution solvents (water:ACN 95:5, 85:15, 75:2, and 50:50\% v/v) 
spectrometer ion source conditions before sample analysis. The parameters were chosen according to the LC flow rate employed, nature of the analytes present in the TM, and their impact on ion formation and transmission. ${ }^{33}$ Spray voltage values of 3.9 and $3.4 \mathrm{kV}$ for ESI+ and 3.6 and $3.1 \mathrm{kV}$ for ESI-, capillary temperatures of 300 and $320{ }^{\circ} \mathrm{C}$, and S-Lens RF voltages levels of 50, 60 and 70 were tested using the TM solution to improve the detection of analytes.

The aim of using the TM for ion source tuning was to improve ion generation and transmission and to decrease unwanted in-source fragmentation of analytes. The decrease in in-source fragmentation may reduce data complexity and thus facilitate data processing and compound annotation. Considering the parameters tested, only the $\mathrm{S}$-Lens RF voltage presented a considerable impact on the peak signal intensity of the TM compounds.

The S-lens is a stacked-ring ion guide responsible for transmitting ions from the ion source to the mass analyzer. ${ }^{34}$ Because the optimal RF voltage depends on the $\mathrm{m} / \mathrm{z}$ to be passed through the $\mathrm{S}$-lens, this value is adjusted according to each precursor mass. The magnitude of the S-lens RF level affects the ions introduced in the MS. Increasing its value will increase the transmission of high $\mathrm{m} / \mathrm{z}$ ions, while decreasing it will favor the transmission of low $\mathrm{m} / \mathrm{z}$ ions. The fragmentation of fragile ions in the S-lens region is also increased due to higher RF levels. Figure 3 clearly shows that fragmentation occurred at the ion source for heptaminol (loss of $18.01 \mathrm{Da}$ (water) from the $\mathrm{m} / \mathrm{z}, 146.1539$ ), and it was more pronounced when the applied RF voltage was higher. The peak area of its $[\mathrm{M}+\mathrm{H}]^{+}$adduct was significantly reduced $(42 \%)$ when the S-lens value was changed from 50 to 70 a.u. Other compounds were less affected by tuning the S-lens. For example, the peak area reduction for benzamide $(\mathrm{m} / \mathrm{z}, 122.0600)$ was only $12 \%$. Conversely, the chlorogenic acid $(\mathrm{m} / \mathrm{z}, 353.0878)$ peak area increased by $29 \%$ by raising the S-lens voltage from 50 to 70 , indicating a higher transmission rate of high $\mathrm{m} / \mathrm{z}$ ions (data not shown).

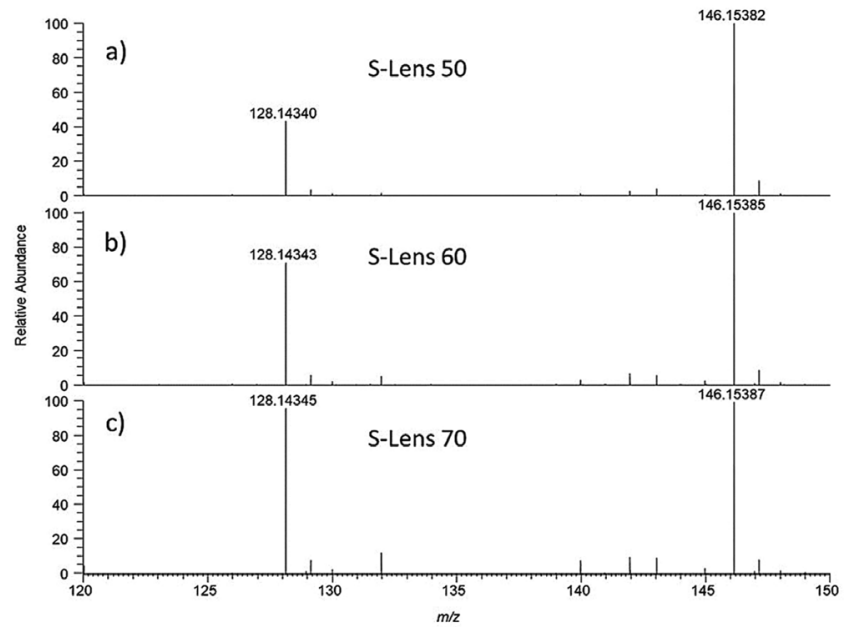

Figure 3. Three levels of S-Lens $R F$ voltages for heptaminol a) 50; $b) 60$ and c) 70 arbitrary units

Although the small number of compounds in the TM does not encompass the complexity of any biological sample used for untargeted metabolomics, these results highlighted the importance of using such a strategy to understand and adequately evaluate the ion source parameters. As in untargeted methods, most of the metabolites are unknown before the analysis; a balance between high ion transmission versus low fragmentation at the ion source must be pursued. Thus, by analyzing the TM solution, the conditions selected to proceed with the samples were spray voltage $3.9 \mathrm{kV}$ (ESI+) and $3.6 \mathrm{kV}$ (ESI-), capillary temperature $320^{\circ} \mathrm{C}$, and the S-lens 60 .

\section{Application of the TM for column performance evaluation}

Column degradation due to deterioration of stationary phase or clogging is a phenomenon expected to occur during its continuous use. Retention time shifts and peak shape distortion should be constantly monitored as signals of column degradation. These effects are most pronounced in large-scale metabolomic studies because of the high number of samples commonly analyzed. Furthermore, sample preparation is kept as simple as possible in untargeted methods, resulting in still very complex samples. Thus, the column performance must be monitored to guarantee that substances with different properties will be consistently eluted across different samples.

In this study, six Syncronis $\mathrm{RP}_{-} \mathrm{C}_{18}$ columns $(50 \mathrm{~mm} \times 2.1 \mathrm{~mm}$, $1.7 \mu \mathrm{m})$ extensively used for urine sample analyses $(>350$ injections each column) were evaluated using the TM solution. Automatic processing of sample injections (ion extraction using $5.0 \mathrm{ppm}$ mass error tolerance, peak integration, and report generation) was performed by the TraceFinder software. Retention times for the TM compounds were compared to those obtained by a brand-new column (same batch and part number, and adequately conditioned before use) as a reference of the expected retention times. The evaluated columns were named $\mathrm{C} 01, \mathrm{C} 02, \mathrm{C} 03, \mathrm{C} 04, \mathrm{C} 05, \mathrm{C} 06$.

Columns C05 and C06 showed increased pressures at initial mobile phase conditions ( 460 bar compared to the average pressure of $340 \mathrm{bar}$ ). The extracted ion chromatograms for the compounds quinic acid $\left(\mathrm{m} / \mathrm{z}\right.$ 191.0561, expected $\left.\mathrm{t}_{\mathrm{R}}=0.35 \mathrm{~min}\right), 3,5$-dinitrobenzoic acid $\left(m / z\right.$ 210.9997, expected $\left.t_{R}=5.55 \mathrm{~min}\right)$, propylhexedrine $\left(\mathrm{m} / \mathrm{z}, 156.1747\right.$, expected $\left.\mathrm{t}_{\mathrm{R}}=6.85 \mathrm{~min}\right)$, and triamcinolone $\left(\mathrm{m} / \mathrm{z}\right.$ 393.1719, expected $\left.\mathrm{t}_{\mathrm{R}}=6.74 \mathrm{~min}\right)$ are shown in supplementary data (Figures $2 \mathrm{~S}$ to $5 \mathrm{~S}$, respectively) for each LC column.

The analyte quinic acid (1) is an example of an early eluting peak due to the presence of hydroxyl groups that interact with the mobile phase by strong hydrogen bond interactions. Because the $\mathrm{C}_{18}$ stationary phase poorly retained this compound, a Gaussian-like peak was observed for all columns. The $\mathrm{C} 01$ column presented the highest $t_{R}$ drift (increased retention) compared to the new one.

Propylhexedrine (7) is a basic compound that eluted at the middle of the analysis and can reveal unwanted secondary interactions. Tailing effects generally occur due to the interactions of the exposed acidic silanol groups from the stationary phase support with basic solutes, causing a loss of separation efficiency. This may ultimately lead to a loss of resolution due to peak tailing and peak integration difficulty (the end of a peak is hard to determine). The extracted ion chromatograms for this compound showed Gaussian-like peak shape only for the columns $\mathrm{C} 02$ and $\mathrm{C} 04$. Front tailing was observed for $\mathrm{C} 05$ and C06 (asymmetry factor, As $=0.8$ and 0.7 , respectively), which could indicate loss of stationary phase and the formation of channels in the column (Figure 3S). Peak splitting was detected only for the $\mathrm{C} 03$, indicating severe column damage.

In conditions that affect acidic compounds, such as stationary phase damage/loss or excess of matrix deposition, 3,5-dinitrobenzoic acid will present a broadened peak and tailing, thus being an indicator of column adsorption effects (Figure $4 \mathrm{~S}$ ). The sample matrix that accumulates at the column inlet may interact with the analytes, usually causing peak broadening and, in extreme cases, peaks to tail into each other. Distorted peaks were observed for 3,5-dinitrobenzoic acid in columns C03, C05, and C06 (the width for these peaks ranged from $0.9 \mathrm{~min}$ for $\mathrm{C} 03$ to $1.7 \mathrm{~min}$ for $\mathrm{C} 05$, while the expected value should be 0.2-0.4 min). The increased pressure for columns C05 and C06 corroborated the suggestion of contamination at the column inlet. Peak splitting was detected again for C03, suggesting that the stationary phase has been irreversibly damaged because of its extensive use. 
Triamcinolone is a C21-steroid carrying four hydroxy substituents. This compound interacts strongly with the $\mathrm{C}_{18}$ external layer bed of the stationary phase through hydrophobic interactions (van der Waals). This solute can also interact with its hydroxyl groups through hydrogen bonding with residual silanols present at the support of the stationary phase surface. This adsorption effect was observed again for column C01 (As = 1.2). It seems that this column has changed its overall surface polarity, probably due to polar or ionized silanol groups on the surface of the support that were irreversibly bound with polar analyte components. Peak splitting and broadening effects were only detected for column $\mathrm{C} 03$, the one that seemed to be the most damaged among the tested columns as the peaks were similarly distorted for nearly all standards.

It was shown that depending on the column conditions, peak integration of distinct substances was strongly and differently affected by column degradation effects. Thus, depending on the purpose of the study to be performed, a column with some altered properties can still be suitable for targeted analysis. The analyst must be confident that specific column conditions will not affect the integration of the target compounds. However, the use of even low damaged columns can lead to irreproducible and erroneous results in untargeted analysis. This highlights the importance of adopting such TM for column performance evaluation. Acceptance criteria for TM analysis in untargeted methods can be assessed by retention time precision $(<2 \%)$, peak area precision ( $\mathrm{CV}$ values $<10 \%)$, mass accuracy tolerance $\left(<5\right.$ ppm for HRMS), and Gaussian-like peak shape. ${ }^{9,11}$

\section{Application of TM in an untargeted metabolomics study}

Various approaches have been described in the literature for ensuring that the results from untargeted methods are valid and trustable, including the use of standard test mixtures (TM), pooled QC samples, and isotopically labeled internal standards (IS) spiked in samples.

In this study, the developed TM was used to assess the suitability of the LC-MS system and the data quality from untargeted metabolomics before performing any multivariate analysis. This study was performed using two RP-C ${ }_{18}$ columns (Syncronis RP-C ${ }_{18}$ columns, $50 \mathrm{~mm} \times 2.1 \mathrm{~mm}, 1.7 \mu \mathrm{m}$ ), named $\mathrm{C} 07$ (considered in good conditions after evaluation with the TM) and C08 (extensively used and with initial signs of degradation after evaluation with the TM).

After a rapid and visual inspection of the TM samples injected at the beginning and the end of each batch in columns $\mathrm{C} 07$ and $\mathrm{C} 08$ (Figures 6S-9S), it was possible to observe a completely different profile, with adequate separation and peak shape for compounds in column $\mathrm{C} 07$ versus peak splitting and signs of column degradation at the end of the batch for column C08. These results indicated that data generated from the untargeted metabolomic analysis in column $\mathrm{C} 08$ were not to be trusted or worth proceeding for multivariate analysis because asymmetric or split-peaks would result in inadequate peak integration and, consequently, data irreproducibility. Nonetheless, the unsupervised classification method PCA and the supervised method PLS-DA were performed.

PCA was done to get an overview of data distribution and the similarities/differences among samples (Figure 4). Quality evaluation of data was assessed by inspecting the pooled QC samples prepared and injected alongside the test samples. The more precise and reproducible the LC-MS analysis, the more tightly the pooled QC samples must be in the PCA space. Although the pooled QC samples were clustered in both PCA models for the two columns tested (Figure 4), indicating that the LC-MS system was suitable for sample analysis, higher dispersion of these samples was observed in
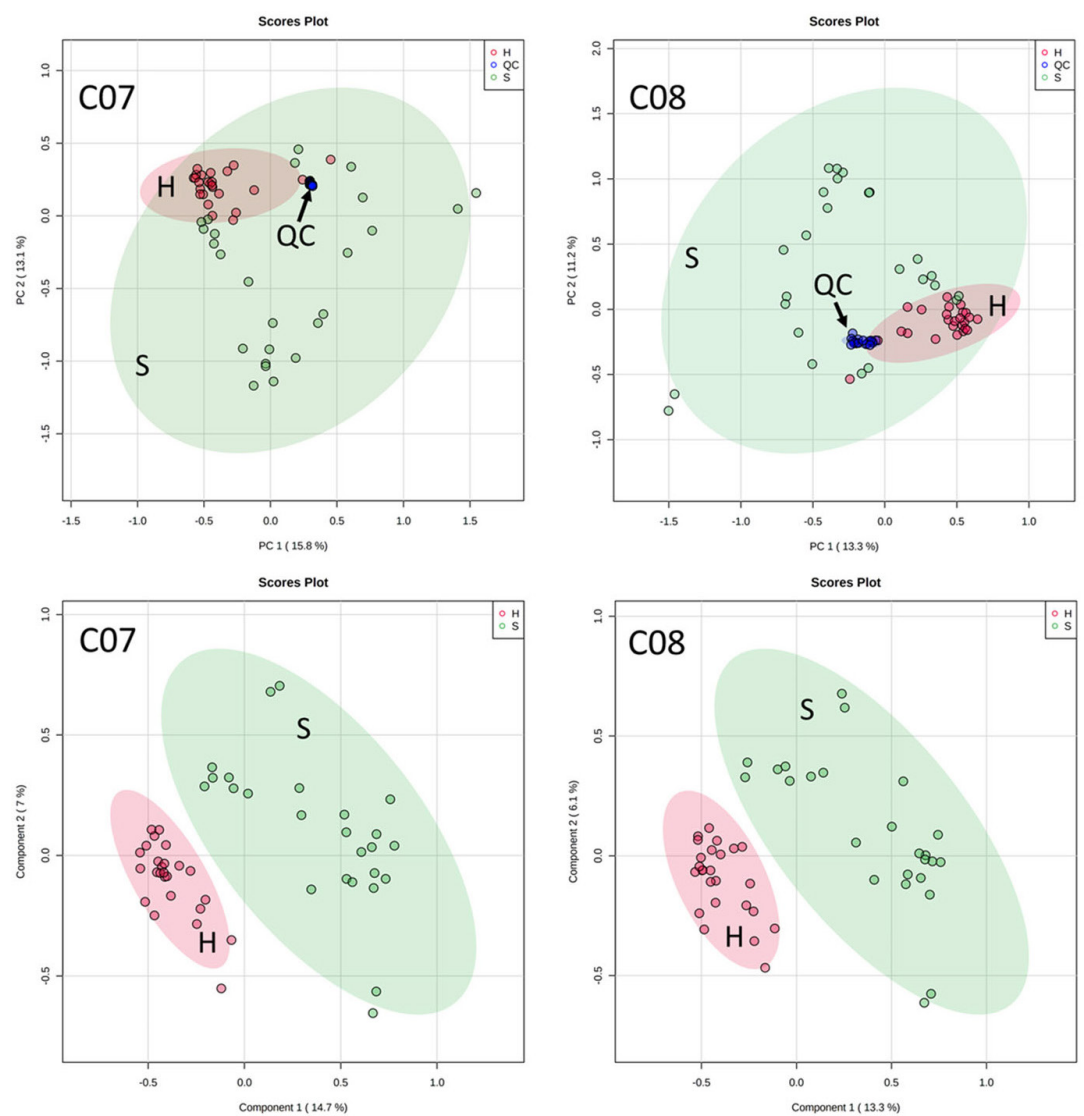

Figure 4. PCA (upper) and PLS-DA (down) plots of the untargeted metabolomic analysis of urine samples by LC-MS for columns C07 (left) and C08 (right). $H=$ healthy volunteers (red); $S=$ suspected of IEM (light green); $Q C=$ pooled $Q C$ samples (dark blue in PCA) 

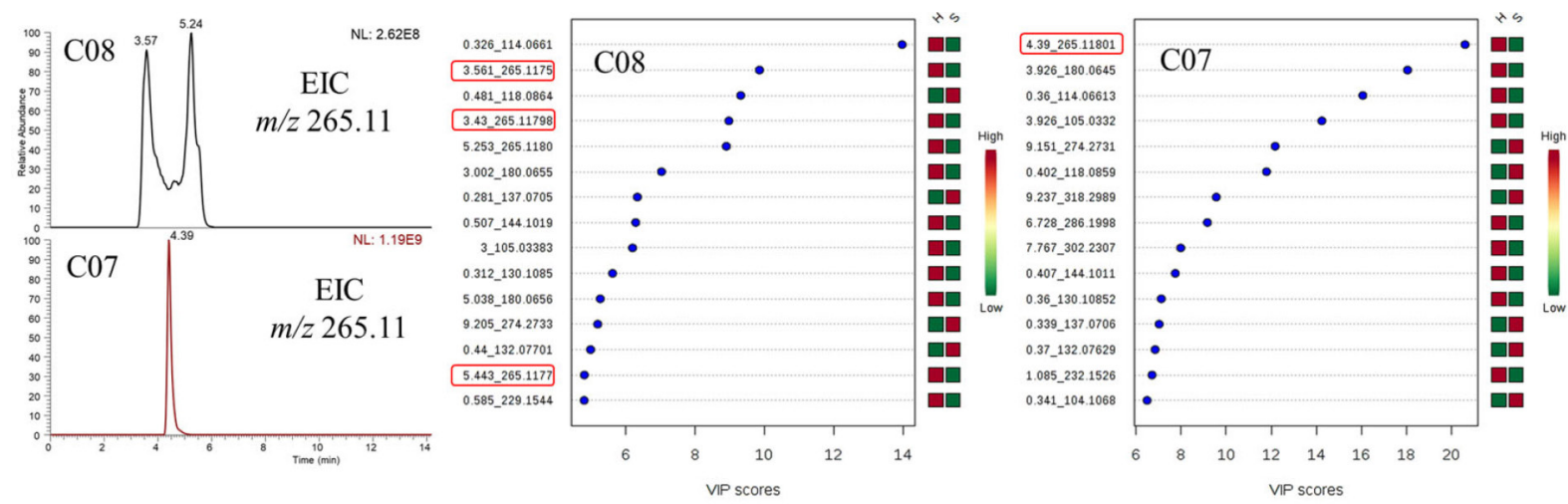

Figure 5. LC-MS extracted ion chromatogram of PLS-DA VIP m/z 265.11 (left) and PLS-DA VIP plots (right) from the untargeted metabolomics of urine samples analyzed in columns C08 and C07. The $\mathrm{m} / z, 265.11$ is highlighted in red in PLS-DA VIP plots

column C08 compared to C07. However, by only a visual inspection of pooled QC samples' dispersion at column C08 and without using a TM or a detailed investigation of the pooled QC samples, ${ }^{11}$ an analyst could erroneously proceed with the statistical analysis in the search for biomarker discovery.

PLS-DA of urine samples in both columns showed a similar profile (Figure 4). The two groups ( $\mathrm{H}=$ healthy volunteers; $\mathrm{S}=$ suspected of IEM) were successfully discriminated. Most of the variables responsible for group discrimination, indicated by the VIP plot (Figure 5), were common for both columns. However, a careful investigation of these variables revealed that three VIP of $\mathrm{m} / \mathrm{z} 265.11$ with a high score from the analysis in column C08 were the same compound. The peak splitting was a result of column degradation during the batch acquisition (Figure 5).

These results showed that although the pooled QC samples are widely used by the metabolomics community to monitor the LC-MS system's precision and stability, without a careful examination of the data, only looking at the pooled QC samples clustering in PCA, it cannot correctly fulfill its function. The analyst may erroneously proceed with the statistical analyses wasting precious time with poor-quality data.

Isotopically labeled IS spiked in samples and pooled QC samples is a common practice in untargeted metabolomics. It is used to evaluate data quality after sample acquisition in a similar way to TM. Moreover, this approach accounts for the sample matrix influence. Considering that the peak shape and $t_{R}$ of some compounds from the TM were not affected or at least less affected than other compounds in columns with different conditions (as previously shown), there is a need to use a reasonable list of IS. However, isotopically labeled IS are usually costly, hampering the use of various compounds spiked in samples and pooled QC samples. Thus, the use of the TM combined with other quality control practices seems a reasonable approach.

\section{CONCLUSIONS}

The TM proved to be a reasonable choice as the first step in untargeted metabolomics method tuning, even having a limited number of compounds that may not directly reflect the complexity of biological samples.

As a system suitability sample, the TM evaluation before sample analysis may easily indicate a system failure, avoiding unnecessary injection (and then reinjection) of a large number of samples commonly employed in untargeted metabolomics. When assessed after sample analysis, it may give information to avoid wasting precious time processing poor-quality data.

The TM was used to monitor the LC-MS system regardless of the biological sample matrix. Its systematic and continuous use over time may allow developing control charts to track the system behavior. This strategy is helpful to verify tendencies and the need to perform early interventions in the analytical system.

Although pooled QC samples are essential for providing a set of data from which repeatability can be assessed throughout the batch, its visual inspection in a PCA space does not directly reflect the chromatographic separation and peak integration quality. The TM analysis as well as the analysis of isotopically labeled IS spiked in samples and pooled QC samples could quickly and effectively track these parameters before further data processing. Thus, a combination of TM, pooled QC samples, and other quality control practices guarantee data reliability in untargeted metabolomic studies.

\section{SUPPLEMENTARY MATERIAL}

Supplementary data (structures and chromatograms) are available free of charge at http://quimicanova.sbq.org.br as PDF file.

\section{ACKNOWLEDGEMENTS}

The authors thank The Brazilian Doping Control Laboratory (LBCD-LADETEC-IQ/UFRJ) for providing some of the chemical standards used in this work. This research was funded by the Conselho Nacional de Desenvolvimento Científico e Tecnológico (CNPq), grant number 161100/2017-1 and Fundação de Amparo à Pesquisa do Estado do Rio de Janeiro - FAPERJ, grant number 010.002501/2019.

\section{REFERENCES}

1. Becker, S.; Kortz, L.; Helmschrodt, C.; Thiery, J.; Ceglarek, U.; J. Chromatogr. B 2012, 883-884, 68.

2. Patti, G. J.; Yanes, G. S. O.; Nat. Rev. Mol. Cell Biol. 2012, 13, 263.

3. Dudzik, D.; Barbas-Bernardos, C.; García, A.; Barbas, C.; J. Pharm. Biomed. Anal. 2018, 147, 149.

4. Gika, H. G.; Theodoridis, G. A.; Wingate, J. E.; Wilson, I. D.; J. Proteome Res. 2007, 6, 3291.

5. Want, E. J.; Wilson, I. D.; Gika, H.; Theodoridis, G.; Plumb, R. S.; Shockcor, J.; Holmes, E.; Nicholson, J. K.; Nat. Protoc. 2010, 5 , 1005.

6. Gika, H. G.; Theodoridis, G. A.; Earll, M.; Wilson, I. D. Bioanalysis 2012, 4, 2239 .

7. Dunn, W. B.; Broadhurst, D.; Begley, P.; Zelena, E.; Francis-Mcintyre, S.; Anderson, N.; Brown, M.; Knowles, J. D.; Halsall, A.; Haselden, J. N.; Nicholls, A. W.; Wilson, I. D.; Kell, D. B.; Goodacre, R.; Nat. Protoc. 2011, 6, 1060

8. Dunn, W. B.; Wilson, I. D.; Nicholls, A. W.; Broadhurst, D.; Bioanalysis 2012, 4, 2249. 
9. Broadhurst, D.; Goodacre, R.; Reinke, S. N.; Kuligowski, J.; Wilson, I. D.; Lewis, M. R.; Dunn, W. B.; Metabolomics 2018, 14, 72.

10. Brunius, C.; Shi, L.; Landberg, R.; Metabolomics 2016, 12, 173.

11. Gika, H. G.; Zisi, C.; Theodoridis, G.; Wilson, I. D.; J. Chromatogr. B 2016, 1008, 15.

12. Engskog, M. K. R.; Haglöf, J.; Arvidsson, T.; Pettersson, C.; Metabolomics 2016, 12, 114.

13. Sangster, T.; Major, H.; Plumb, R.; Wilson, A. J.; Wilson, I. D.; Analyst 2006, 131, 1075 .

14. Want, E. J.; Masson, P.; Michopoulos, F.; Wilson, I. D.; Theodoridis, G.; Plumb, R. S.; Shockcor, J.; Loftus, N.; Holmes, E.; Nicholson, J. K.; Nat. Protoc. 2013, 8, 17.

15. Beger, R. D.; Dunn, W. B.; Bandukwala, A.; Bethan, B.; Broadhurst, D.; Clish, C. B.; Dasari, S.; Derr, L.; Evans, A.; Fischer, S.; Flynn, T.; Hartung, T.; Herrington, D.; Higashi, R.; Hsu, P. C.; Jones, C.; Kachman, M.; Karuso, H.; Kruppa, G.; Lippa, K.; Maruvada, P.; Mosley, J.; Ntai, I.; O’Donovan, C.; Playdon, M.; Raftery, D.; Shaughnessy, D.; Souza, A.; Spaeder, T.; Spalholz, B.; Tayyari, F.; Ubhi, B.; Verma, M.; Walk, T.; Wilson, I.; Witkin, K.; Bearden, D. W.; Zanetti, K. A.; Metabolomics 2019, 15, 4.

16. Tang, L.; Fitch, W. L.; Alexander, M. S.; Dolan, J.; Anal. Chem. 2000, 72,5211 .

17. Shu, L.; Julien, L.; Tidswell, P.; Goetzinger, W.; J. Comb. Chem. 2006, 8,820 .

18. Argmann, C. A.; Houten, S. M.; Zhu, J.; Schadt, E. E.; Cell Metab. 2016, $23,13$.

19. Scalco, F. B.; Oliveira, M. L. C.; Simoni, R. E.; Aquino Neto, F. R.; J. Braz. Chem. Soc. 2014, 25, 1914.

20. Saudubray, J. M.; Garcia-Cazorla, À.; Pediatr. Clin. North Am. 2018, 65, 179.

21. Jacob, M.; Malkawi, A.; Albast, N.; Al Bougha, S.; Lopata, A.; Dasouki, M.; Abdel Rahman, A. M. A.; Anal. Chim. Acta 2018, 1025, 141.

22. Coene, K. L. M.; Kluijtmans, L. A. J.; van der Heeft, E.; Engelke, U. F. H.; de Boer, S.; Hoegen, B.; Kwast, H. J. T.; van de Vorst, M.; Huigen,
M. C. D. G.; Keularts, I. M. L. W.; Schreuder, M. F.; van Karnebeek, C. D. M.; Wortmann, S. B.; de Vries, M. C.; Janssen, M. C. H.; Gilissen, C.; Engel, J.; Wevers, R. A.; J. Inherit. Metab. Dis. 2018, 41, 337.

23. Miller, M. J.; Kennedy, A. D.; Eckhart, A. D.; Burrage, L. C.; Wulff, J. E.; Miller, L. A. D.; Milburn, M. V.; Ryals, J. A.; Beaudet, A. L.; Sun, Q.; Sutton, V. R.; Elsea, S. H.; J. Inherit. Metab. Dis. 2015, 38, 1029.

24. Blaženović, I.; Kind, T.; Sa, M. R.; Ji, J.; Vaniya, A.; Wancewicz, B.; Roberts, B. S.; Torbašinović, H.; Lee, T.; Mehta, S. S.; Showalter, M. R.; Song, H.; Kwok, J.; Jahn, D.; Kim, J.; Fiehn, O.; Anal. Chem. 2019, 91, 2155

25. Helfer, A. G.; Michely, J. A.; Weber, A. A.; Meyer, M. R.; Maurer, H H.; Anal. Chim. Acta 2015, 891, 221.

26. Gevi, F.; Belardo, A.; Zolla, L. Biochim. Biophys. Acta Mol. Basis Dis. 2020, 1866, 165859.

27. Ruan, Y.; Zheng, J.; Ren, Y.; Tang, J.; Li, J.; Li, D.; Food Funct. 2019, $10,2471$.

28. Mendes Resende, J. V.; de Sá, N. M. D.; de Oliveira, M. T. L.; Lopes, R. C.; Garrett, R.; Borges, R. M.; Phytochem. Lett. 2020, 36, 99.

29. van der Hooft, J. J. J.; Padmanabhan, S.; Burgess, K. E. V.; Barrett, M. P.; Metabolomics 2016, 12, 125.

30. Tsugawa, H.; Cajka, T.; Kind, T.; Ma, Y.; Higgins, B.; Ikeda, K.; Kanazawa, M.; Vandergheynst, J.; Fiehn, O.; Arita, M. Nat. Methods 2015, 12,523 .

31. Chong, J.; Soufan, O.; Li, C.; Caraus, I.; Li, S.; Bourque, G.; Wishart, D. S.; Xia, J.; Nucleic Acids Res. 2018, 46, W486.

32. Mutton, I.; Boughtflower, B.; Taylor, N.; Brooke, D.; J. Chromatogr. A 2011, $1218,3711$.

33. Sardela, V. F.; Martucci, M. E. P.; de Araújo, A. L. D.; Leal, E. C.; Oliveira, D. S.; Carneiro, G. R. A.; Deventer, K.; Van Eenoo, P.; Pereira, H. M. G.; Aquino Neto, F. R.; J. Mass Spectrom. 2018, 53, 476.

34. Olsen, J. V.; Schwartz, J. C.; Griep-Raming, J.; Nielsen, M. L.; Damoc, E.; Denisov, E.; Lange, O.; Remes, P.; Taylor, D.; Splendore, M.; Wouters, E. R.; Senko, M.; Makarov, A.; Mann, M.; Horning, S.; Mol. Cell. Proteomics 2009, 8, 2759. 